\title{
TITLE: A tool to predict progression of non-alcoholic fatty liver disease in severely obese patients
}

Pierre Bauvin, 1,

Claire Delacôte, $1^{*}$,

Guillaume Lassailly, 1,2*

Line Carolle Ntandja Wandji, 2,

Viviane Gnemmi, 3,

Flavien Dautrecque, 2,

Alexandre Louvet, 1,2,

Robert Caiazzo, 4,

Violeta Raverdy, 4,

Emmanuelle Leteurtre, 5,

François Pattou, 4,

Sylvie Deuffic-Burban, 1,6†,

Philippe Mathurin, 1,2†

${ }^{*}, \dagger$ equal contribution

1 Univ. Lille, Inserm, CHU Lille, U1286 - INFINITE - Institute for Translational Research in Inflammation, F-59000 Lille, France

2 Hôpital Claude Huriez, Services Maladies de l'Appareil Digestif, CHRU Lille, 59000 Lille, France.

3 Univ. Lille, CHU Lille, Inserm UMR-S 1172, Department of Pathology, Centre de Biologie Pathologie, 59000 Lille, France

4 Univ. Lille, Inserm, CHU Lille, U1190 - EGID, 59000 Lille, France

5 Univ. Lille, Inserm, CHU Lille, UMR-S 1172 - JPARC - Jean-Pierre Aubert Research Center, F59000 Lille, France

6 Université de Paris, IAME, INSERM, F-75018 Paris, France 


\section{Contact information:}

Philippe Mathurin,

Hôpital Huriez - Service Maladies de l'Appareil Digestif - CHRU Lille, Rue Michel Polonovski - 59037

LILLE CEDEX

Phone : +33 3204455 97, Philippe.MATHURIN@CHRU-LILLE.FR

Sylvie Deuffic-Burban

Université de Paris - IAME - Inserm, 16 rue Henri Huchard, 75018 Paris, France

sylvie.burban@inserm.fr

Word count: 4678

Number of figures \& tables: 7

\section{Abbreviations}

NAFLD: Non-alcoholic Fatty Liver Disease

NASH: Non-alcoholic Steatohepatitis

BMI: Body Mass Index

NL: Normal Liver

\section{ACKNOWLEDGEMENTS}

This work is supported by the French government, managed by the National Research Agency (ANR) under the Future Investment Program (PIA2) with the reference ANR-16-RHUS-0006_PreciNASH. 


\section{ABSTRACT}

Background\&Aims: Severely obese patients are a growing population at risk of non-alcoholic fatty liver disease (NAFLD). Considering the increasing burden, a predictive tool of NAFLD progression would be of interest. Our objective is to provide a tool allowing general practitioners to identify and refer the patients most at risk, and specialists to estimate disease progression and adapt the therapeutic strategy.

Methods: This predictive tool is based on a Markov model simulating steatosis, fibrosis and nonalcoholic steatohepatitis (NASH) evolution. This model was developped from data of 1,801 severely obese, bariatric surgery candidates, with histological assessment, integrating duration of exposure to risk factors. It is then able to predict current disease severity in the absence of assessment, and future cirrhosis risk based on current stage.

Results: The model quantifies the impact of sex, body-mass index at 20, diabetes, age of overweight onset, on progression. For example, for 40 -year-old severely obese patients seen by the general practitioners: $(A)$ non-diabetic woman overweight at 20 , and (B) diabetic man overweight at 10 , without disease assessment, the model predicts their current risk to have NASH or F3-F4: for (A) $5.7 \%$ and $0.6 \%$, for (B) $16.1 \%$ and $10.0 \%$, respectively. If those patients have been diagnosed $\mathrm{F} 2$ by the specialist, the model predicts the 5 -year cirrhosis risk: $1.8 \%$ in the absence of $\mathrm{NASH}$ and $6.0 \%$ in its presence for $(A), 10.3 \%$ and $26.7 \%$, respectively, for $(B)$.

Conclusions: This model provides a decision-making tool to predict the risk of liver disease that could help manage severely obese patients.

\section{Keywords:}

$\mathrm{NASH}$; fibrosis; Markov model; disease progression; risk factors 


\section{LAY SUMMARY}

A predictive tool would be of great interest to manage the burden of non-alcoholic fatty liver disease (NAFLD) and in particular to identify and refer patients at the highest risk as well as estimate disease progression and adapt therapeutic strategies. We have developed this predictive tool for severely obese patients based on the history of candidates for bariatric surgery, including data on the history of overweight, obesity and diabetes. The model describes the NAFLD progression from the overweight onset and is able to predict the current disease stages, with or without information from histological or non-invasive tests, as well as progression at 5 years. 


\section{INTRODUCTION}

Non-alcoholic fatty liver disease (NAFLD), which is associated with an increased risk of liver-related morbidity and mortality, ${ }^{1-3}$ is a growing public health issue and has become one of the main etiologies in adults waiting for liver transplantation..$^{4,5}$ The disease burden increased along with the prevalence of obesity and metabolic syndrome, its two main causes. ${ }^{6,7}$

The histopathological spectrum of NAFLD includes isolated steatosis, non-alcoholic steatohepatitis (NASH) and the different stages of fibrosis. ${ }^{18,9}$ Natural history studies have shown that NAFLD patients with NASH disclose significantly higher risk of fibrosis progression than those with isolated steatosis. ${ }^{10-13}$ Although fibrosis progression is slower in patients without NASH, development of cirrhosis occurs in patients with simple steatosis. ${ }^{12,14}$ Indeed, in NAFLD F3-F4 patients, simple steatosis is observed in around $20 \%$ and NASH in $80 \%$ of cases. ${ }^{12,14}$ Many cofactors are also associated with disease progression: genetic variants, metabolic syndrome, diabetes, sex and age. ${ }^{15-}$ ${ }^{18}$ However, despite improved understanding of the natural history of NAFLD, data are still lacking on the role of the duration of exposure, an important component in chronic disease. In particular, how does the duration of exposure to overweight, obesity and/or diabetes impacts the severity of NAFLD and its evolution.

Severely obese candidates for bariatric surgery are a population of interest to analyse the impact of obesity and diabetes history on NAFLD. ${ }^{14}$ Indeed, these patients present a wide heterogeneity in NAFLD severity, history of obesity, diabetes, and metabolic syndrome. The policy of our centre consists in performing systematic liver biopsy at surgery, and the use of questionnaires on the past history of obesity and diabetes, two crucial factors in the setting of NAFLD. Such data collection is an opportunity to provide more insights into NAFLD progression by exploring the relationship between histological features, clinical characteristics and duration of exposure to obesity and/or diabetes. An indicator of liver risk that integrates all of this relevant information is an attractive approach to identify patients at high risk of disease progression.

Bariatric surgery induces sustained weight-loss, improves metabolic comorbidities, frequently resolves NASH (80\% of cases) and improves fibrosis (60\% of cases). ${ }^{19}$ When considering the number of severely obese with NAFLD, there is a need to prioritize those who would benefit most from 
surgery. Such approach requires to optimize patients' path from general practitioners to specialized centres, and improve the decision-making process for patients already addressed in the centre. For the general practitioners, the availability of a user-friendly tool identifying those with high risk of advanced fibrosis or rapid disease progression is attractive. In specialized centres, baseline assessment of fibrosis is more and more performed by non-invasive methods such as dosage of biomarkers or measurement of liver stiffness. ${ }^{20} \mathrm{~A}$ tool integrating this partial information to predict the disease progression would have added value. Mathematical modelling is a method of choice for the development of such a tool. ${ }^{21,22}$

The aims of our study were to quantify the progression to steatosis, fibrosis and $\mathrm{NASH}$, identify and evaluate the impact of independent disease progression cofactors and provide a tool predicting disease progression based on a modelling approach. 


\section{MATERIALS AND METHODS}

We developed a Markov model to describe NAFLD progression among severely obese, using preoperative histological and clinical data from 1,801 candidates for bariatric surgery from the "Lille Bariatric Cohort". ${ }^{23}$ The model considered disease progression from the initial stage of the normal liver through cirrhosis. It assessed the transition probabilities from one stage to another as well as the effects of covariates on these transitions, estimated from the observed data. The resulting model predicts the current disease stage of an individual, as well as its progression in the future, taking into account the duration of being overweight, obese and individual characteristics. A web-based tool is available for both the evaluation of disease severity, and the prediction of disease progression at https://ille-model.shinyapps.io/deployedapp/.

\section{Markov model}

The progression of NAFLD to compensated cirrhosis was modelled according to the severity of fibrosis and the development of NASH through the 10 following stages of liver disease (Figure 1): normal liver (NL), simple steatosis with F0 to F3 fibrosis, NASH with F0 to F3 fibrosis, and cirrhosis. ${ }^{12,14}$ The progression of steatosis, fibrosis or NASH are estimated as the result of the combination of progression and regression, as in other modelling studies of NAFLD. ${ }^{24}$ For more details, please refer to the Supporting Information.

We assumed that all patients had a NL when they initially became overweight, and then were at-risk of NAFLD. We assessed this risk from one available biopsy from each patient, which represented the stage of progression over the exposure time.

\section{Study population and histological analysis}

The "Lille Bariatric Cohort" consists of 2,735 severely or morbidly obese patients referred for bariatric surgery between 1994 and 2018 and prospectively included, as described in Lassailly et al (details in Supporting Information, section "Inclusion criteria"). ${ }^{19}$ Moreover, $12(0.4 \%)$ patients were candidates for bariatric surgery and had a liver biopsy, but didn't undergo surgery because of serious conditions. 
They were added to the cohort to be closer to a real-life distribution of disease severity. All patients were invited to follow a nutrition program before surgery.

A standardized questionnaire was prospectively presented to each patient in the cohort at inclusion to specifically assess the history of overweight and obesity, recording the following information: period when weight gain occurred, age of onset of obesity and body mass index (BMI) at a 20 years old. Patients were asked about their overweight history by reporting the period when weight gain occurred as: "before the age of 7" referring to kindergarten/preschool ("école maternelle") which corresponds to children up to 6 years old; "between age 7 and puberty" referring to primary school ("école primaire") which corresponds to children aged 7 to 11 ; "during puberty" which refers to secondary school ("collège") and corresponds to adolescents aged 12 to 15 , and "post puberty", i.e. after secondary school "collège". We used these data to infer the age of overweight onset; for more details, please refer to the Supporting Information, section "Exposure time". The presence of diabetes was defined by ADA criteria. ${ }^{25}$ Diabetic patients were asked the age of diagnosis of diabetes. Liver biopsies were systematically planned during the surgical procedure. Diagnosis of NASH was performed by the presence of hepatocellular steatosis, ballooning/clarification and inflammation, the well-recognized histological features of NASH. ${ }^{26}$ Severity of the necroinflammatory activity was assessed using the NAS score. ${ }^{27}$ Fibrosis was characterized by both the Metavir and Kleiner scores. ${ }^{27,28}$ In the baseline analysis, we used the Metavir score for developing the model. An alternative analysis using the Kleiner score is presented in the Supporting Information section "Sensitivity analysis".

Four hundred and seventy-three patients were excluded due to past history of alcohol, age under 18, histological data lacking or because they were referred from other medical centre. Four hundred seventy-three of the remaining 2,274 patients (20.8\%) were excluded because essential clinical data were missing for the glycaemia assessment or the onset of weight gain (flow-chart in Figure 2). Finally, 411 of the 1,801 remaining patients $(22.8 \%)$ couldn't recall their weight at $20(309 / 1,801)$ and/or the age at the diagnosis of diabetes (148/600 patients with diabetes). To include these patients in the model, the missing BMI at 20 years old and/or age at the onset of diabetes were imputed based on the following variables: sex, period of onset of overweight, age, BMI and the presence of diabetes at the biopsy. We used the widely used, ${ }^{29}$ predictive mean matching method. ${ }^{30,31}$ 
Informed written consent was obtained for all patients and the study was performed in accordance with the Declaration of Helsinki. The ethics committee approved the cohort and the study was supported by grants from the government and the French Ministry of Health (PHRC). After legal revision, new approval was obtained in 2006 (no. CP06/49, NCT01129297)

\section{Covariates and exposure time}

The effects of independent covariates were evaluated to take into account the heterogeneity of disease progression: sex, period of onset of overweight, BMI category at 20 years old and the presence of diabetes. We used data on period of onset of overweight to infer the age of overweight onset. Exposure time is the duration of exposure to overweight/obese (BMI $\geq 25)$, when the patient is at-risk of developing NAFLD. It was calculated as the difference between the age at biopsy and the age of overweight onset (details in Supporting Information, section "Exposure time").

\section{Procedure}

The parameters of progression are estimated by the maximum likelihood estimates method,,$^{32}$ using the biopsy data from the "Lille Bariatric Cohort". The underlying methodology has been previously validated, 22,33-35 and is described in the Supporting Information, section "Estimation of the parameters".

To assess the internal validity of the model, the total cohort was randomly divided into a training cohort $(n=1,207 ; 67 \%)$ and a testing cohort $(n=594 ; 33 \%)$. The training cohort was used to estimate the parameters. The testing cohort was used to evaluate the internal validity of the model, by calculating the global error score, i.e. the weighted mean of the relative differences between the number of individuals at each stage observed at the date of biopsy and the number predicted by the model throughout the period of exposure (see details in Supporting Information, section "Model evaluation"). For further validation, we used k-fold cross-validation ( $k=10)$ described in the Supporting Information. 
Once the parameters are estimated, the model can predict the current state of the liver state for any hypothetical patient, by simulating the disease progression from the age s/he became overweight. Moreover, it can also able predict the 5-year risk of disease progression, based on the current assessment of fibrosis. We chose to present the 5-year risk of the development of cirrhosis in two contexts: 1) with partial information provided by non-invasive methods, i.e. only the stage of fibrosis without information on NASH status (in this case, NASH status had to be estimated by the model); and 2) with complete information provided by biopsy i.e. NASH status and stage of fibrosis.

The technical aspects of model building, testing and use are further detailed in the Supporting Information section "Methodological appendix". To gauge model uncertainty all of these results were provided with a plausible interval obtained from a probabilistic sensitivity analysis. ${ }^{36}$

All analyses were performed by PB with the R-software (https://www.R-project.org/) version 3.3.3 and the msm package, under the supervision of SDB and PM. ${ }^{37}$ 


\section{RESULTS}

\section{Patient characteristics}

The characteristics of the population are summarized in Table 1, separately for the study cohort $(n=1,801)$ and the two randomly selected subsets (training cohort, $n=1,207$ and testing cohort, $\mathrm{n}=594$ ). In the study cohort, at biopsy $74.5 \%$ were women, severely or morbidly obese (mean BMI \pm $\mathrm{SD}$ of $47.6 \pm 8 \mathrm{~kg} / \mathrm{m}^{2}$ ), with mean age of $41.2 \pm 11$ years old. Histological NASH was diagnosed in $183 / 1,801(10.2 \%)$ cases, and cirrhosis in 27 (1.5\%) patients.

\section{Adequacy with data}

The error scores for each histological stage (presented in Supporting Table 1) show that the model adequately reproduces disease progression in all the stages of NAFLD. Good accuracy is confirmed by a global error score of $4.5 \%$ in the training cohort and $8.8 \%$ in the testing cohort. If we consider error rates $>15 \%$, the model overestimates the number of patients with F2-steatosis (11 predicted versus 7 observed), with F3-steatosis (5 predicted versus 4 observed) and with F3-NASH (9 predicted versus 6 observed), while it underestimates the number of patients with F2-NASH (8 predicted, versus 11 observed). It accurately predicts the number of patients with cirrhosis (9 predicted vs 9 observed).

\section{Transition rates}

Table 2 summarizes the ten baseline transition rates and adjusted hazard ratios (HR) of the covariates estimated from the training cohort. Baseline transition rates correspond to a patient profile with the following characteristics: women who became overweight after puberty, with a BMI of 25 $\mathrm{kg} / \mathrm{m}^{2}$ at 20 years old and without diabetes. For example, with the above characteristics, the baseline transition rate and $95 \%$ confidence interval $(\mathrm{Cl})$ from NL to $\mathrm{F} 0$-steatosis is $7.72 \%(6.85 \%-8.56 \%)$, meaning that around 8 out of 100 of these patients with NL develop steatosis per year. The Markov model provides transition rates specific to each patient's profile from baseline rates and adjusted HR. 
For example, patients with the above characteristics, starting overweight at 5 years old have reduced yearly transition rates toward steatosis $(5.25 \%, 95 \% \mathrm{Cl}=4.53 \%-6.07 \%)$ than patients with onset of overweight at 15 , i.e. during puberty $(6.39 \%, 95 \% \mathrm{Cl}=5.55 \%-7.36 \%)$. However, as expected, the former have a greater probability of steatosis at $20(48.32 \%)$ than the latter $(26.13 \%)$, due to the fact that their duration of exposure is longer ( 15 years versus 5 years)

The patients with the fastest disease progression are diabetic men, who became overweight during the post-puberty period, and who are class II or III obese $(\mathrm{BMI}>30)$ at 20 . These patients have an annual progression rate from steatosis to $\mathrm{NASH}$ of $2.17 \%(95 \% \mathrm{Cl}: 1.68 \%-2.77 \%)$, compared to $0.61 \%$ (95\% Cl: $0.52 \%-0.73 \%$ ) in standard patients (women, non-diabetic, who became overweight during the post-puberty period and who have a BMI at age 20 indicating that they are overweight).

Those results are relatively unchanged among the different sensitivity analysis (details in Supporting Information, section"Sensitivity analysis", and Supporting Tables 2-7).

\section{Evaluation of disease severity}

By simulating disease progression from its starting point, this model evaluates the probability of being in a disease stage based on individual characteristics. Figure 3 shows the model-predicted risks according to three patient profiles. First, 40-year-old non-diabetic women who became overweight at 20 (i.e., during the post-puberty period, with a BMI at age 20 indicating that they are overweight and with 20 years of exposure to the disease) have a probability of $21.4 \%$ (plausible interval: $17.8 \%$ $24.9 \%)$ to still have a NL, $72.8 \%(68.8 \%-76.8 \%)$ to have steatosis, $5.7 \%(4.5 \%-7.1 \%)$ to have $\mathrm{NASH}$ and $0.1 \%(0.1 \%-0.2 \%)$ to have cirrhosis (Figure $3 \mathrm{~A})$. Furthermore, there is a $0.6 \%(0.4 \%-$ $1.1 \%)$ probability of F3-F4 fibrosis (0.2\% steatosis-F3-F4, 0.4\% NASH-F3-F4).

Second, patients with the above characteristics who have been diabetic since the age of 35 , have a $13.3 \%(11.2 \%-17.5 \%)$ probability of having a NL, $78.0 \%(74.0 \%-80.3 \%)$ of having steatosis, $8.4 \%$ $(6.9 \%-9.7 \%) \mathrm{NASH}$ and $0.3 \%(0.2 \%-0.5 \%)$ cirrhosis (Figure 3B). There is a $1.7 \%(1.2 \%-2.1 \%)$ probability of having F3-F4 fibrosis (0.6\% steatosis-F3-F4, 1.1\% NASH-F3-F4) in these patients. 
Finally, 40-year-old men who have been overweight at 10, obese class II at 20 and diabetic at 35 have a probability of $2.7 \%(1.8 \%-4.6 \%)$ of having a NL, $77.6 \%(72.8 \%-81.0 \%)$ of having steatosis, $16.1 \%(13.3 \%-19.8 \%)$ NASH and $3.6 \%(2.4 \%-5.5 \%)$ cirrhosis (Figure $3 \mathrm{C})$. These patients have a $10.0 \%(7.1 \%-13.4 \%)$ probability of having F3-F4 fibrosis (3.3\% steatosis-F3-F4, 6.7\% NASH-F3-F4).

\section{Prediction of 5-year disease progression}

The present model predicts the cumulative risk of cirrhosis at 5 years based on non-invasive assessment of baseline fibrosis, or biopsy. Figure 4 shows this risk according to different severities of fibrosis evaluated by non-invasive methods in 40 -year-old non-diabetic women who became overweight at 20 years old. The five-year risk of cirrhosis in these patients with baseline NL or <F2 fibrosis is $0.02 \%$ (plausible interval: $0.02 \%-0.04 \%)$, ranging from $0.006 \%(0.003 \%-0.02 \%)$ in the absence of NASH to $0.3 \%(0.2 \%-0.6 \%)$ in those with $\mathrm{NASH}$ at baseline (Figure $4 \mathrm{~A})$. The five-year risk in the same patients with baseline $\geq \mathrm{F} 2$ fibrosis is $12.0 \%(10.2 \%-16.6 \%)$, ranging from $7.2 \%$ $(4.8 \%-13.5 \%)$ in the absence of NASH to $17.8 \%(15.1 \%-21.2 \%)$ in the presence of NASH (Figure 4B).

When the disease stage is assessed by liver biopsy, providing a precise stage of fibrosis and the presence or absence of $\mathrm{NASH}$, the model also predicts the probability of reaching each disease stage according to this baseline histological information. For example, previous patients with F2-steatosis have a 5 -year risk of cirrhosis of $1.8 \%(0.7 \%-5.2 \%)$, compared to $6.0 \%(3.9 \%-9.1 \%)$ in F2-NASH patients and $21.5 \%(17.3 \%-26.5 \%)$ in F3-NASH patients (Figure 5). In more severe profiles (men who become overweight at 10, obese class II at 20 and diabetic at 35), F2 patients without NASH have a 5 -year risk of cirrhosis of $10.3 \%(4.3 \%-25.5 \%)$, compared to $26.7 \%(20.0 \%-34.2 \%)$ in the presence of $\mathrm{NASH}$, and $47.0 \%(40.4 \%-53.8 \%)$ in $\mathrm{F} 3-\mathrm{NASH}$ patients (not shown). 


\section{DISCUSSION}

Our model provides a robust tool for predicting the course of disease in severely obese patients. The novelty of our work is that all the model parameters are estimated from preoperative liver biopsies and individual characteristics from the Lille Bariatric Cohort. Moreover, the model takes into account the global overweight history, including the age of overweight onset and BMI at 20, to assess the relationship between the progression of NAFLD and the duration of exposure to being overweight, obese and with diabetes. We modelled the patient's average disease history, by considering that, as stated elsewhere, candidates for bariatric surgery could not achieve long-term weight loss, ${ }^{38}$ while taking into account that they could achieve temporary regression of steatosis, fibrosis or NASH. Another key aspect is that the resulting predictions can be obtained in the absence of information about the current stage of fibrosis or after integrating disease stage determined by either liver biopsy or non-invasive methods. Our work shows that patients with similar baseline characteristics may have marked differences in their risk of progression due to diverse past histories of obesity and diabetes.

As previously reported, ${ }^{39,40}$ the model shows that NASH accelerates disease progression with variations according to stage of fibrosis. The model also weights the relative impact of different independent cofactors such as diabetes, male sex, BMI at 20 years old and the age category of overweight onset. As examples, our study confirm the impact of diabetes and male sex, as previously reported, ${ }^{41,42}$ leading to an increase of the progression rate by $202 \%$ in a diabetic woman compared to a non-diabetic woman, and $291 \%$ between a diabetic man and a non-diabetic woman, other factors being equal. An interesting insight is that age of overweight onset appears as an important predictive factor: in two patients with the same histological diagnosis at the same age, the patient who became overweight later is identified with faster disease progression, all other factors being equal.

Our model is available as a web-based tool (https://lille-model.shinyapps.io/deployedapp/). It can be used to improve the care pathway of severely obese patients, and to support the decision for bariatric surgery according to the risk of the progression of liver disease. However, it is important to note that the model does not consider other important metabolic comorbidities. It is now possible to identify which severely obese patients should be referred to specialists during the clinical evaluation based on the prediction of the stage of liver disease. Specialists working in the bariatric surgery unit can now 
select patients with rapid disease progression whatever their baseline fibrosis, in whom delaying bariatric surgery would be deleterious to the liver. Specialists working in the bariatric surgery unit can now select patients with rapid disease progression whatever their baseline fibrosis, in whom delaying bariatric surgery would be deleterious to the liver. For example, 50-year-old non-diabetic women, who became overweight at 20, diagnosed with a F3-NASH have a lower 5-year risk of cirrhosis $(21.5 \%)$ than 50-year-old diabetic men, who become overweight at 15 years old, obese class I at 20 and diabetic at 35, and diagnosed with F2-NASH (25.8\%). However, this approach opens up the unsolved issue of what is the appropriate cut-off risk of disease progression to undergo bariatric surgery. Such cut-off should be proposed by experts and would need to be estimated in a second step in prospective studies.

The present study has certain limitations. First, the model may be subject to selection bias, because the training is based on data from severely obese patients. Thus, it should only be used in these patients. Moreover, external validation was not available. Although the model was internally validated using a split into the training-testing cohorts, as well as a cross-validation procedure described in the Supporting Information, future validation is required to strengthen these conclusions. This shows the importance of recording the patients' history of obesity, which is often lacking in bariatric surgery cohorts. Second, our approach may have resulted in an undersampling of cirrhosis, as this event is often a contraindication for bariatric surgery. However, we decided to include patients with cirrhosis who were candidates but who were not selected for surgery, to limit this bias. As a result, the prevalence of compensated cirrhosis was $1.8 \%$ in NAFLD patients, which is coherent with the prevalence observed in other, non-bariatric cohorts of NAFLD patients with different demographics. ${ }^{43}$ Patients with decompensated cirrhosis were not referred for bariatric surgery in the first place, but this group is outside the scope of this study. Third, because of the absence of prospective, repeated liver biopsies, the model was based on one biopsy from living patients at the time of surgery, and assuming that patients had a normal liver when they became overweight. The only way to obtain an unbiased evaluation of the natural history of disease would be sequential biopsies from unselected patients and in the absence of treatment, which to our knowledge are not available, due to ethical reasons. In order to test the potential immortal time bias, as all patients had to survive to inform the model, a model incorporating background mortality from the literature was also tested in sensitivity analysis and have led to similar results. ${ }^{38}$ Fourth, our study could be limited by a memory bias. 
Indeed, even if the data on the history of obesity was collected during specific face-to-face interviews, we cannot rule out errors in the patients' memories of their history of overweight/obesity, especially if it occurred during childhood. As the age of onset is a key variable, we decided to exclude patients with this missing information. Moreover, a sensitivity analysis was performed to test the impact of the recall bias using a simplified age of onset of overweight and, again, similar results were obtained. Fifth, we imputed the missing covariates "BMI at 20 years old" and "age of diagnosis of diabetes" in 411 cases. We used the predictive mean matching method which is the recommended approach and produced the least biased estimates. ${ }^{44,45}$ Such imputation seems to not affect the resulting model, as the results were relatively unchanged after exclusion of the patients with imputed data, as described in the Supporting Information.

In conclusion, our model can simulate and predict the progression of NAFLD from its onset to cirrhosis in severely obese patients. It shows that taking into account the duration of exposure to key risk factors is essential. This model could help in the management of NAFLD in severely obese patients, by providing a decision-making tool to help refer and select patients for bariatric surgery based on predicted liver status. 


\section{COMPETING INTERESTS}

None: Pierre Bauvin, Claire Delacôte, Flavien Dautrecque, Line Carole Ntandja Wandji, Viviane Gnemmi, Alexandre Louvet, Emmanuelle Leteurtre.

Guillaume Lassailly has received lecture fees from Gilead and Novartis.

Sylvie Deuffic-Burban has received consultancy honoraria from Intercept, and lecture fees from Abbvie and Gilead.

Philippe Mathurin was paid speaking at symposiums for Abbvie, Gilead Sciences, Ipsen, Eisai, MSD, Bayer Healthcare pharmaceutical companies. He is member of the French boards of experts in hepatology for Abbvie, MSD, Gilead Sciences, Ipsen, Eisal and Bayer Healthcare pharmaceutical companies. He is consulting for the MSD, Ipsen, Eisal, Abbvie, Sanofi, Gilead Sciences, Servier, Bayer Healthcare.

The others authors did not answer about their potential conflicts of interest at the time of submission.

\section{AUTHOR CONTRIBUTIONS}

Philippe Mathurin and Sylvie Deuffic-Burban contributed to the study idea and conceptualization. Pierre Bauvin, Claire Delacôte, Guillaume Lassailly, Philippe Mathurin and Sylvie Deuffic-Burban contributed to the methodology, the conception and design of the analysis. All authors contributed to data curation, acquisition and interpretation. Pierre Bauvin performed the formal analysis, devised, programmed and ran the model. Pierre Bauvin, Claire Delacôte, Guillaume Lassailly, Line Carolle Ntandja Wandji, Sylvie Deuffic-Burban and Philippe Mathurin drafted the manuscript. All authors revised it critically for important intellectual content and approved the final version to be published. All authors agree to be accountable for all aspects of the work in ensuring that questions related to the accuracy or integrity of any part of the work are appropriately investigated and resolved. 


\section{FUNDING}

This work is supported by the French government, managed by the National Research Agency (ANR) under the Future Investment Program (PIA2) with the reference ANR-16-RHUS-0006_PreciNASH. 


\section{REFERENCES}

1. Chalasani N, Younossi Z, Lavine JE, et al. The diagnosis and management of nonalcoholic fatty liver disease: practice guideline by the American Gastroenterological Association, American Association for the Study of Liver Diseases, and American College of Gastroenterology. Gastroenterology. 2012;142(7):1592-1609.

2. Paradis V, Zalinski S, Chelbi E, et al. Hepatocellular carcinomas in patients with metabolic syndrome often develop without significant liver fibrosis: a pathological analysis. Hepatology. 2009;49(3):851-859.

3. Povsic M, Wong OY, Perry R, Bottomley J. A Structured Literature Review of the Epidemiology and Disease Burden of Non-Alcoholic Steatohepatitis (NASH). $A d v$ Ther. 2019;36(7):1574-1594.

4. Wong RJ, Cheung R, Ahmed A. Nonalcoholic steatohepatitis is the most rapidly growing indication for liver transplantation in patients with hepatocellular carcinoma in the U.S. Hepatology. 2014;59(6):2188-2195.

5. Marcellin P, Kutala BK. Liver diseases: A major, neglected global public health problem requiring urgent actions and large-scale screening. Liver Int. 2018;38 Suppl $1: 2-6$.

6. Lazo M, Hernaez R, Eberhardt MS, et al. Prevalence of nonalcoholic fatty liver disease in the United States: the Third National Health and Nutrition Examination Survey, 1988-1994. Am J Epidemiol. 2013;178(1):38-45.

7. Kotronen A, Westerbacka J, Bergholm R, Pietilainen KH, Yki-Jarvinen H. Liver fat in the metabolic syndrome. J Clin Endocrinol Metab. 2007;92(9):3490-3497.

8. Neuschwander-Tetri BA, Caldwell SH. Nonalcoholic steatohepatitis: summary of an AASLD Single Topic Conference. Hepatology. 2003;37(5):1202-1219.

9. Starley BQ, Calcagno CJ, Harrison SA. Nonalcoholic fatty liver disease and hepatocellular carcinoma: a weighty connection. Hepatology. 2010;51(5):1820-1832.

10. Ekstedt M, Franzen LE, Mathiesen UL, et al. Long-term follow-up of patients with NAFLD and elevated liver enzymes. Hepatology. 2006;44(4):865-873.

11. Younossi ZM, Stepanova M, Rafiq N, et al. Pathologic criteria for nonalcoholic steatohepatitis: interprotocol agreement and ability to predict liver-related mortality. Hepatology. 2011;53(6):1874-1882.

12. Angulo P, Kleiner DE, Dam-Larsen S, et al. Liver Fibrosis, but No Other Histologic Features, Is Associated With Long-term Outcomes of Patients With Nonalcoholic Fatty Liver Disease. Gastroenterology. 2015;149(2):389-397 e310.

13. Loomba R, Chalasani N. The Hierarchical Model of NAFLD: Prognostic Significance of Histologic Features in NASH. Gastroenterology. 2015;149(2):278-281. 
14. Bedossa P, Tordjman J, Aron-Wisnewsky J, et al. Systematic review of bariatric surgery liver biopsies clarifies the natural history of liver disease in patients with severe obesity. Gut. 2017;66(9):1688-1696.

15. Speliotes EK, Butler JL, Palmer CD, et al. PNPLA3 variants specifically confer increased risk for histologic nonalcoholic fatty liver disease but not metabolic disease. Hepatology. 2010;52(3):904-912.

16. Valenti L, Al-Serri A, Daly AK, et al. Homozygosity for the patatin-like phospholipase-3/adiponutrin I148M polymorphism influences liver fibrosis in patients with nonalcoholic fatty liver disease. Hepatology. 2010;51(4):1209-1217.

17. Adams LA, Sanderson S, Lindor KD, Angulo P. The histological course of nonalcoholic fatty liver disease: a longitudinal study of 103 patients with sequential liver biopsies. J Hepatol. 2005;42(1):132-138.

18. Dixon JB, Bhathal PS, O'Brien PE. Nonalcoholic fatty liver disease: predictors of nonalcoholic steatohepatitis and liver fibrosis in the severely obese. Gastroenterology. 2001;121(1):91-100.

19. Lassailly G, Caiazzo R, Buob D, et al. Bariatric Surgery Reduces Features of Nonalcoholic Steatohepatitis in Morbidly Obese Patients. Gastroenterology. 2015;149(2):379-388; quiz e315-376.

20. Castera L. Invasive and non-invasive methods for the assessment of fibrosis and disease progression in chronic liver disease. Best Pract Res Clin Gastroenterol. 2011;25(2):291-303.

21. Beck JR, Pauker SG. The Markov process in medical prognosis. Med Decis Making. 1983;3(4):419-458.

22. Deuffic-Burban S, Poynard T, Valleron AJ. Quantification of fibrosis progression in patients with chronic hepatitis $\mathrm{C}$ using a Markov model. J Viral Hepat. 2002;9(2):114-122.

23. Marshall G, Guo W, Jones RH. MARKOV: a computer program for multi-state Markov models with covariables. Comput Methods Programs Biomed. 1995;47(2):147-156.

24. Estes C, Razavi H, Loomba R, Younossi Z, Sanyal AJ. Modeling the epidemic of nonalcoholic fatty liver disease demonstrates an exponential increase in burden of disease. Hepatology. 2018;67(1):123-133.

25. Expert Committee on the Diagnosis Classification of Diabetes Mellitus. Report of the expert committee on the diagnosis and classification of diabetes mellitus. Diabetes Care. 2003;26 Suppl 1:S5-20.

26. Brunt EM, Janney CG, Di Bisceglie AM, Neuschwander-Tetri BA, Bacon BR. Nonalcoholic steatohepatitis: a proposal for grading and staging the histological lesions. Am J Gastroenterol. 1999;94(9):2467-2474. 
27. Kleiner DE, Brunt EM, Van Natta M, et al. Design and validation of a histological scoring system for nonalcoholic fatty liver disease. Hepatology. 2005;41(6):13131321.

28. Bedossa P, Poynard T. An algorithm for the grading of activity in chronic hepatitis C. The METAVIR Cooperative Study Group. Hepatology. 1996;24(2):289-293.

29. van Buuren S, Groothuis-Oudshoorn K. mice: Multivariate Imputation by Chained Equations in R. 2011. 2011;45(3):67.

30. Little RJA. Missing-Data Adjustments in Large Surveys. Journal of Business \& Economic Statistics. 1988;6(3):287-296.

31. Rubin DB. Statistical Matching Using File Concatenation With Adjusted Weights and Multiple Imputations. Journal of Business \& Economic Statistics. 1986;4(1):87-94.

32. Jackson CH, Sharples LD, Thompson SG, Duffy SW, Couto E. Multistate Markov models for disease progression with classification error. Journal of the Royal Statistical Society: Series D (The Statistician). 2003;52(2):193-209.

33. Delacote C, Bauvin P, Louvet A, et al. A Model to Identify Heavy Drinkers at High Risk for Liver Disease Progression. Clin Gastroenterol Hepatol. 2020.

34. Longini IM, Jr., Clark WS, Byers RH, et al. Statistical analysis of the stages of HIV infection using a Markov model. Stat Med. 1989;8(7):831-843.

35. Marshall G, Jones RH. Multi-state models and diabetic retinopathy. Stat Med. 1995;14(18):1975-1983.

36. Zhang Y, Wu H, Denton BT, Wilson JR, Lobo JM. Probabilistic sensitivity analysis on Markov models with uncertain transition probabilities: an application in evaluating treatment decisions for type 2 diabetes. Health Care Manag Sci. 2019;22(1):34-52.

37. Jackson CH. Multi-State Models for Panel Data: The msm Package for R. Journal of Statistical Software. Journal of Statistical Software. 2011;38(8):1-29.

38. Sjostrom L, Narbro K, Sjostrom CD, et al. Effects of bariatric surgery on mortality in Swedish obese subjects. N Engl J Med. 2007;357(8):741-752.

39. Singh S, Allen AM, Wang Z, Prokop LJ, Murad MH, Loomba R. Fibrosis progression in nonalcoholic fatty liver vs nonalcoholic steatohepatitis: a systematic review and meta-analysis of paired-biopsy studies. Clin Gastroenterol Hepatol. 2015;13(4):643654 e641-649; quiz e639-640.

40. Matteoni CA, Younossi ZM, Gramlich T, Boparai N, Liu YC, McCullough AJ. Nonalcoholic fatty liver disease: a spectrum of clinical and pathological severity. Gastroenterology. 1999;116(6):1413-1419.

41. Pang Y, Kartsonaki C, Turnbull I, et al. Diabetes, Plasma Glucose, and Incidence of Fatty Liver, Cirrhosis, and Liver Cancer: A Prospective Study of 0.5 Million People. Hepatology. 2018;68(4):1308-1318. 
42. Lonardo A, Nascimbeni F, Ballestri S, et al. Sex Differences in Nonalcoholic Fatty Liver Disease: State of the Art and Identification of Research Gaps. Hepatology. 2019;70(4):1457-1469.

43. Hagstrom H, Nasr P, Ekstedt M, et al. Fibrosis stage but not NASH predicts mortality and time to development of severe liver disease in biopsy-proven NAFLD. J Hepatol. 2017;67(6):1265-1273.

44. Marshall A, Altman DG, Holder RL. Comparison of imputation methods for handling missing covariate data when fitting a Cox proportional hazards model: a resampling study. BMC Med Res Methodol. 2010;10:112.

45. Marshall A, Altman DG, Royston P, Holder RL. Comparison of techniques for handling missing covariate data within prognostic modelling studies: a simulation study. BMC Med Res Methodol. 2010;10:7. 
Table 1 - Clinical, biological, and histological characteristics of patients at biopsy and before

\begin{tabular}{|c|c|c|c|c|}
\hline & $\begin{array}{l}\text { All } \\
(n=1801)\end{array}$ & $\begin{array}{l}\text { Training } \\
\text { cohort } \\
(n=1207)\end{array}$ & $\begin{array}{l}\text { Testing } \\
\text { cohort } \\
(n=594)\end{array}$ & P-value* \\
\hline Female sex, n (\%) & $1341(74.5)$ & $898(74.4)$ & $443(74.6)$ & 0.98 \\
\hline \multicolumn{5}{|l|}{ At biopsy } \\
\hline Age, mean \pm SD & $41.2( \pm 11)$ & $41.3( \pm 12)$ & $41.0( \pm 11)$ & 0.52 \\
\hline $\mathrm{BMI}\left(\mathrm{kg} / \mathrm{m}^{2}\right)$, mean $\pm \mathrm{SD}$ & $47.6( \pm 8)$ & $47.3( \pm 8)$ & $48.2( \pm 8)$ & 0.03 \\
\hline Diabetes, n (\%) & $600(33.3)$ & $411(34.1)$ & $189(31.8)$ & 0.37 \\
\hline Steatosis, n (\%) & $1527(84.8)$ & $1021(84.6)$ & $506(85.2)$ & 0.99 \\
\hline $\mathrm{NASH}, \mathrm{n}(\%)$ & $183(10.2)$ & $121(10.0)$ & $62(10.4)$ & 0.85 \\
\hline \multicolumn{5}{|l|}{ Fibrosis (Metavir), n (\%) } \\
\hline Fo & $1359(75.5)$ & $902(74.7)$ & $457(76.9)$ & 0.33 \\
\hline $\mathrm{F} 1$ & $321(17.8)$ & $221(18.3)$ & $100(16.8)$ & 0.48 \\
\hline $\mathrm{F} 2$ & $58(3.2)$ & $40(3.3)$ & $18(3.0)$ & 0.86 \\
\hline F3 & $36(2.0)$ & $26(2.2)$ & $10(1.7)$ & 0.62 \\
\hline $\mathrm{F} 4$ & $27(1.5)$ & $18(1.5)$ & $9(1.5)$ & 0.99 \\
\hline
\end{tabular}

History of overweight/obesity :

Onset of overweight, n (\%)

Before 7 years old

$395(21.9) \quad 265(22.0) \quad 130(21.9) \quad 0.99$ 
Between 7 years old and puberty

$$
332(18.4) \quad 225(18.6) \quad 107(18.0) \quad 0.80
$$

$\begin{array}{llll}\text { At puberty } \quad 368(20.4) & 230(19.1) & 138(23.2) & 0.05\end{array}$

$\begin{array}{lllll}\text { Post puberty } & 706(39.2) & 487(40.3) & 219(36.9) & 0.17\end{array}$

Age at the onset of obesity, mean $\pm \mathrm{SD}$

$22.1( \pm 9) \quad 22.1( \pm 9) \quad 20.9( \pm 9) \quad 0.32$

Age at the onset of diabetes

among patients with diabetes, mean

$41.0( \pm 9) \quad 41.2( \pm 9) \quad 39.9( \pm 9) \quad 0.10$ $\pm \mathrm{SD}$

BMI at 20 years old, $n(\%)$

Normal weight $\left(\mathrm{BMI}<25 \mathrm{~kg} / \mathrm{m}^{2}\right) \quad 459(25.5 \%) \quad 324(26.8 \%) \quad 135(22.7 \%) \quad 0.07$

Overweight $(25 \leq \mathrm{BMI}<30$

$468(26.0 \%) \quad 326(27.0 \%) \quad 142(23.9 \%) \quad 0.18$ $\left.\mathrm{kg} / \mathrm{m}^{2}\right)$

Obese class I $(30 \leq \mathrm{BMI}<35$ $\left.\mathrm{kg} / \mathrm{m}^{2}\right)$

$392(21.8 \%) \quad 245(20.3 \%) \quad 147(24.7 \%) \quad 0.04$

Obese class II \& III (BMI $\geq 35$ $\left.\mathrm{kg} / \mathrm{m}^{2}\right)$

$482(26.8 \%) \quad 312(25.8 \%) \quad 170(28.6 \%) \quad 0.23$

* as the test of difference between training and testing cohorts, using Pearson's Chi-squared test for categorical data, and Welch T-test for continuous data. 
Table 2 - Estimated baseline yearly transition rates for a standard patient, and adjusted hazard ratios of the covariates, impacting these transition rates. A standard patient is a woman without diabetes, who became overweight during the post-puberty period and have a BMI at age 20 indicating that they are overweight .

Baseline transition rates

Normal liver $\rightarrow$ F0 with steatosis

$\mathrm{F} 0 \rightarrow \mathrm{F} 1$, with steatosis

$\mathrm{F} 1 \rightarrow \mathrm{F} 2$, with steatosis

$\mathrm{F} 2 \rightarrow \mathrm{F} 3$, with steatosis

F3 with steatosis $\rightarrow$ cirrhosis

Steatosis $\rightarrow \mathrm{NASH}$, regardless of fibrosis

$\mathrm{F} 0 \rightarrow \mathrm{F} 1$, with $\mathrm{NASH}$

$\mathrm{F} 1 \rightarrow \mathrm{F} 2$, with $\mathrm{NASH}$

$\mathrm{F} 2 \rightarrow \mathrm{F} 3$, with $\mathrm{NASH}$

F3 with $\mathrm{NASH} \rightarrow$ cirrhosis

Hazard ratios

Male sex

Age at onset overweight
$7.72 \%$

$6.85 \%-8.56 \%$

$1.50 \%$

$1.30 \%-1.74 \%$

$1.35 \%$

$0.96 \%-1.83 \%$

$4.93 \%$

$2.87 \%-8.41 \%$

$3.25 \%$

$1.20 \%-8.68 \%$

$0.61 \%$

0.52\%-0.73\%

$7.35 \%$

$5.19 \%-10.10 \%$

$8.37 \%$

$13.35 \%$

$4.84 \%$

$3.77 \%-6.2 \%$

$5.77 \%-12.34 \%$

$10.36 \%-17.15 \%$

1.44

1.35-1.55

$0.59-0.78$ 
Between age 7 and puberty

During puberty

Post puberty

$\mathrm{BMI}$ at 20 years old

Normal weight

Overweight

Obese class I

Obese class II \& III

Presence of type 2 diabetes
0.74

0.83

$0.72-0.96$

(ref)

1

(ref)

1.06

0.95-1.19

1.12

0.98-1.29

1.28

$1.09-1.51$

2.02

$1.74-2.35$ 


\section{LEGEND OF FIGURES}

Figure 1 - Markov model of compensated non-alcoholic fatty liver disease.

Figure 2 - Flow-chart.

Figure 3 - Expected risk of being in one of the stages of the disease, based on individual characteristics, for A) 40 -year-old women patients who became overweight at 20 , and without diabetes, B) 40 year-old women patients who became overweight at 20 and with diabetes since age 35, and C) 40 year-old men patients who became overweight at 10, obese class II at 20 and with diabetes since age 35 .

Figure 4 - Cumulated risk of cirrhosis at 5 years, for 40 year-old women patients who became overweight at $\mathbf{2 0}$ and without diabetes, according to current stage assessed by a non-invasive test: (A) patients with NL or <F2 fibrosis, (B) patients with $\geq F 2$ fibrosis. The dashed line provides a weighted risk estimated without information on NASH status, taking into account the likelihood of having NASH at current evaluation or within 5 years, evaluated by the model.

Figure 5 - Cumulated risk of cirrhosis at 5 years, for 40 year-old women patients who became overweight of 20 and without diabetes, according to current stage assessed by liver biopsy: normal liver, F2-steatosis, F2-NASH, F3-steatosis, F3-NASH. 